\title{
Two-frame 3D particle tracking
}

\author{
Francisco Pereira ${ }^{1}$, Heinrich Stüer ${ }^{2}$, Emilio C Graff $^{2}$ \\ and Morteza Gharib ${ }^{2}$ \\ ${ }^{1}$ INSEAN-Italian Ship Model Basin, Rome, Italy \\ ${ }^{2}$ Graduate Aeronautical Laboratories, California Institute of Technology, Pasadena, \\ CA 91125 , USA \\ E-mail: f.pereira@insean.it
}

Received 3 December 2005, in final form 14 March 2006

Published 7 June 2006

Online at stacks.iop.org/MST/17/1680

\begin{abstract}
A whole-field three-dimensional (3D) particle tracking velocimetry (PTV) tool for diagnostics in fluid mechanics is presented. Specifically, it is demonstrated why and when PTV is the natural choice in 3D applications compared to particle image velocimetry (PIV). Three different tracking methods are investigated, namely the nearest neighbour, the neural network and the relaxation method. In order to demonstrate the use of PTV for 3D applications, the selected tracking schemes are implemented for use with the defocusing digital particle image velocimetry (DDPIV) technique. The performance of the tracking algorithms is evaluated based on synthetic 3D information. Furthermore, the potential benefit of a merging between the PIV and PTV approaches is explored within the DDPIV framework. The results show that the relaxation tracking method is the most robust and efficient, while the combined PIV/PTV analysis brings significant improvements solely with the neural network scheme. In terms of errors, PTV is found to be more sensitive to particle reconstruction errors than the DDPIV cross-correlation analysis.
\end{abstract}

Keywords: three-dimensional, particle tracking, two-frame, defocusing, DDPIV

\section{Introduction}

Cross-correlation-based techniques are widely used in twodimensional (2D) particle image velocimetry (PIV) to analyse image data. These techniques are usually based on greylevel images and generally do not need to determine particle coordinates (Willert and Gharib 1991, Keane and Adrian 1992). Another class of algorithms determining velocity vectors operate on particle coordinates and are called particle tracking velocimetry (PTV) methods (Racca and Dewey 1988, Baek and Lee 1996, Ohmi and Li 2000). Which method one uses depends strongly on the experimental set-up and flow conditions. For instance, if the particle density is high and/or the velocity gradients across a cross-correlation interrogation window are small, then PIV will be the method of choice. On the other hand, if the particles are sparsely distributed in the observation volume, one would prefer PTV.

PIV is the preferred method in $2 \mathrm{D}$ measurement applications, for it does not require us to detect the particle centres. For the three-dimensional (3D) case, almost all successful methods rely on a preprocessing step where the coordinates of every particle image are measured in order to calculate the $3 \mathrm{D}$ coordinates in space. One exception is the so-called holographic PIV method (Barnhart et al 1994). In the following, we would like to illustrate why the PTV method is the natural choice in 3D applications. Although the particle density in image space is in the range of PIV (Adrian 1991), the particle density in physical space is not. This is simply because the particle images are integrated over the whole depth of view on the $2 \mathrm{D}$ image. For example, the mean particle distance, say $d_{0}$, for imaging a square light sheet of side length $c$ or a cube with the same side length varies according to $d_{0,3 \mathrm{D}} \approx d_{0,2 \mathrm{D}} \cdot N_{0}^{1 / 6}$ where $N_{0}$ is the number of particles. For $N_{0} \approx 400$, the mean distance has already tripled in the 3D case compared to the 2D one. Additionally, when small vortices with locally strong velocity gradients are present across the interrogation region, PTV methods will resolve the corresponding vectors of changing magnitude, whereas conventional PIV will yield an averaged vector with lower accuracy as a consequence of the spread correlation 
displacement peak. Consequently, PTV vector fields also inherit a higher spatial resolution than PIV.

The PTV method tracks individual particle images in consecutive image frames and computes the directionally resolved vector for each matched particle. However, the PTV measurements contain individual velocity errors which are mostly related to the estimate of the particle centroids in an image frame. Therefore, it is desirable to have measurements where the particles travel a longer distance than in PIV. For high density seeded flows, this makes tracking difficult and a trade-off between particle density and imaging delay has to be found.

This trade-off depends, of course, on the tracking method used. In conventional PTV, most methods use three or four consecutive flow images (multi-frame methods) for accurate velocity measurements because they are either based on the minimization of Lagrangian acceleration, on the change of Lagrangian acceleration, or on the minimization of length and angle variation of particle tracks between image frames (Kasagi and Nishino 1991, Malik et al 1993, Wernet and Pline 1993). If we want to resolve small time scales and work with double pulsed lasers, these techniques are not applicable as we then need two-frame tracking. Two-frame tracking methods with rather different approaches have been proposed to solve the correspondence problem occurring in particle tracking. For instance, the concept of cluster matching, as opposed to particle matching, has been used by Okamoto et al (1995). Cost functions and genetics-inspired algorithms, such as the neural networks proposed by Grant and Pan (1995) and Labonté (1999), have been found to perform well in the presence of unpaired particles. The relaxation method (Baek and Lee 1996, Ohmi and Li 2000) is based on matching probabilities between the first and second frames, and has proved to be simple and reliable. Very recently, Ruhnau et al (2005) have successfully introduced a computer vision-derived variational approach for PTV, where the whole velocity field is estimated by minimization of a functional composed of a discrete matching term and of a continuous smoothness term. Though this novel approach is shown to yield slightly better results than the most up-to-date schemes (i.e. relaxation), further investigation is needed since the reported comparative evaluation is very limited and restricted to a $2 \mathrm{D}$ flow case.

In the following, we evaluate the applicability of some of these algorithms to the 3D space by means of the defocusing digital particle image velocimetry (DDPIV) technique (Pereira and Gharib 2002). DDPIV is particularly suited for tracking implementation as it requires, by concept, 3D particle identification prior to any velocity calculation. Furthermore, we seek to improve the performance of these selected algorithms using information on the 3D flow field. Specifically, we explore the possibility of using the 3D spatial cross-correlation method, as implemented in DDPIV, to improve the reliability of the tracking schemes presented in this work. This is in some sense in the spirit of the superresolution 2D PIV method introduced by Keane et al (1995) and further developed by Shan and Gharib (1998) and Stitou and Riethmuller (2001).

This paper is concerned with the evaluation of two-frame tracking methods for use in 3D applications. Three tracking schemes, namely the nearest neighbour, the neural network and the relaxation algorithms, are implemented into DDPIV as our preferred $3 \mathrm{D}$ measurement and evaluation tool. By means of simulated data sets reproducing a simple Burgers' vortex and a complex 3D flow, the performance of these three different tracking techniques is evaluated, in terms of tracking efficiency, processing time and velocity errors. A sample application to an actual flow is finally presented.

\section{Two-frame particle tracking}

\subsection{Principles of tracking methods}

Let us assume that the coordinates of particles are given in space and for two consecutive time steps (frames) $t_{0}$ and $t_{1}$. Thus, we are given $N$ coordinates $\left\{\mathbf{x}_{i}=\left(x_{i}^{1}, x_{i}^{2}, x_{i}^{3}\right) \in t_{0}, i=\right.$ $1, \ldots, N\}$, and $M$ coordinates $\left\{\mathbf{y}_{j}=\left(y_{j}^{1}, y_{j}^{2}, y_{j}^{3}\right) \in t_{1}, j=\right.$ $1, \ldots, M\}$. The goal of the tracking procedure is to select the correct link for the same particle from one time step to the next one. Obviously, this can only be a trajectory with a maximum likelihood for specific criteria. The three essential tracking principles can be summarized as follows:

- Estimate the position of the selected particle in the next (search) frame at $t_{1}$.

- Delimit a neighbourhood volume centred on the expected location within which the particle in the search frame is likely to be found.

- Apply suitable (physically based) sorting criteria to determine the most likely track if there exists more than one possible option.

These principles are applicable to any flow regime under examination. The present PTV is designed for turbulent measurements as well as for laminar ones, but can be extended to any tracking task in which moving targets need to be tracked in time.

Once the corresponding particle link $l_{i, j}$ for coordinate $\mathbf{x}_{i}$ from the first frame to the coordinate $\mathbf{y}_{j}$ in the second frame is determined, the velocity $\mathbf{u}\left(\mathbf{x}_{i, j}\right)$ can be estimated by the following first-order finite difference method,

$$
\mathbf{u}_{i, j}=\frac{\mathbf{y}_{j}-\mathbf{x}_{i}}{\Delta t}
$$

where $\Delta t$ is the time difference between the two frames. Additionally, we shift the resulting velocity vector linearly to

$$
\mathbf{x}_{i, j}=\frac{\mathbf{x}_{i}+\mathbf{y}_{j}}{2} .
$$

Note that this procedure does not make equation (1) secondorder accurate because of the linear shift in equation (2).

\subsection{Tracking parameter}

In this subsection, we would like to define some general parameter upon which the success of tracking depends.

We shall define the particle number density $\rho_{0}$ and the void fraction $\alpha_{0}$ by

$$
\rho_{0}=\frac{\int \zeta(a) \mathrm{d} a}{V_{0}}, \quad \alpha_{0}=\frac{\int \zeta(a) v(a) \mathrm{d} a}{V_{0}} .
$$

Here $\zeta(a)$ is the probability density function of particles with radius $a, V_{0}$ is the observation volume, and $v(a)$ is the sphere volume associated with radius $a$. The number of particles 
within the observation volume $V_{0}$ is simply given by

$$
N_{0}=\rho_{0} \cdot V_{0}=\int \zeta(a) \mathrm{d} a .
$$

We also define the probability $p(r)$ of finding a neighbour particle at a distance $r$ by

$$
p(r)=\delta\left(r-d_{0}\right)= \begin{cases}1 & \text { if } r=d_{0} \\ 0 & \text { otherwise }\end{cases}
$$

where $d_{0}$ is the mean particle distance and $\delta$ is the Dirac operator. The mean distances $d_{0,2 \mathrm{D}}$ and $d_{0,3 \mathrm{D}}$, corresponding to the respective probabilities of finding one single particle within a disc and within a sphere, are given after integration of equation (5) by

$$
d_{0,2 \mathrm{D}}=\sqrt{\frac{1}{\pi \rho_{0,2 \mathrm{D}}}}, \quad d_{0,3 \mathrm{D}}=\sqrt[3]{\frac{3}{4 \pi \rho_{0,3 \mathrm{D}}}} .
$$

Therefore, for a cubic domain and a square area, both with side length $c$ and containing the same number $N_{0}$ of particles, we have

$$
\frac{d_{0,3 \mathrm{D}}}{d_{0,2 \mathrm{D}}} \approx 1.099 \cdot N_{0}^{\frac{1}{6}}
$$

The mean distance between particles in the cubic domain doubles for $N_{0}=36$ and triples for $N_{0}=412$ with respect to the planar case.

With $|\mathbf{u}|_{\max }$ being the maximum velocity in the flow domain and $|\mathbf{x}|_{\max }=|\mathbf{u}|_{\max } \cdot \Delta t$ the maximum displacement of a particle, we define the following 3D tracking parameter,

$$
\Phi=\frac{d_{0}}{|\mathbf{x}|_{\max }}
$$

where $d_{0} \equiv d_{0,3 \mathrm{D}}$ as defined in equation (6).

Generally, the success of tracking depends on the probability of finding only one or a small number of particles within the search volume. By using equation (6) and the maximum radius $R_{\mathrm{S}}$ of the search volume, we can define (Malik et al 1993) the parameter $p$ such that

$$
p=\frac{d_{0}}{R_{\mathrm{s}}}
$$

If $p \gg 1$, then the probability of finding only one particle in the search volume is high and tracking is easy and reliable, whereas for $p \ll 1$, successful tracking is unlikely if not impossible. Note that even for a global $p \gg 1$, the local $p$ can be much smaller than 1, in particular if the particle distribution within the volume $V_{0}$ is not homogeneous. Here, and unlike other authors (Malik et al 1993, Baek and Lee 1996), we make an additional distinction between equations (8) and (9). As a matter of fact, equation (8) is identical to equation (9) only if the search volume is centred on the particle at time $t_{0}$.

Our goal is to introduce, as in multi-frame tracking methods, a first estimate of the particle position in $t_{1}$ to delimit the search volume for higher success of tracking and thus speed up the computation. For the case of $p<1$, we tested three different sorting criteria (or tracking methods) which will be explained, together with the estimate of the search volume, in the following subsections.

\subsection{Search volume and neighbourhood volume}

One of the goals of PTV is to delimit and place the search volume such that one or only a small number of matchable candidates exist. A valid radius for the search volume in a turbulent flow would be the maximum deviation distance a particle can travel. This can be estimated using the RMS fluctuation velocities $\mathbf{u}^{\prime}$,

$$
R_{\mathrm{s}}=3 \cdot\left|\mathbf{u}^{\prime}\right| \cdot \Delta t .
$$

This estimate is not the only choice for $R_{\mathrm{s}}$. Malik and Dracos (1993) estimated

$$
R_{\mathrm{s}}=\frac{1}{3} d_{0}
$$

which is also the appropriate choice for laminar flow cases.

The neighbourhood radius $R_{\mathrm{n}}$ defines a correlation radius within which the velocity field is highly correlated. The Taylor micro-scale, $\lambda_{\mathrm{T}}$, is often used in experimental and theoretical work for this purpose. Hence, we define $R_{\mathrm{n}}$ to be a fraction of $\lambda_{\mathrm{T}}$ :

$$
R_{\mathrm{n}}=F_{\mathrm{n}} \cdot \lambda_{\mathrm{T}} .
$$

If the observation volume $V$ is much larger than $\lambda_{\mathrm{T}}$, then $F_{\mathrm{n}} \approx 1$ is the correct choice. For small observation volumes, such that $V^{1 / 3} \approx \lambda_{\mathrm{T}}$ (or smaller), then all velocities in the volume will be correlated. In this situation, we do not need to consider all velocity vectors and only an arbitrary small fraction, $F_{\mathrm{n}} \ll 1$, can be taken in order to limit the number of particles within the correlation neighbourhood.

The position $\tilde{\mathbf{y}}_{i}$ of the search volume in the second frame relative to the particle coordinate $\mathbf{x}_{i}$ in the first frame can be determined by the convection velocity $\mathbf{u}_{\mathrm{c}, i}$ of the particle $i$ :

$$
\tilde{\mathbf{y}}_{i}=\mathbf{x}_{i}+\mathbf{u}_{\mathrm{c}, i} \cdot \Delta t
$$

\subsection{Estimating the local convection velocity}

Obviously, the more we know about the flow field, the better the position and size of the search volume can be estimated, and the tracking method will perform more successfully. If the mean velocity, of say a turbulent flow, is a priori known then this can be used for $\mathbf{u}_{\mathrm{c}, i}$. Malik et al (1993) and Stüer (1999) have developed refined search volume estimates that improve the tracking efficiency. For two-dimensional (2D) flow fields, the local convection velocity is usually estimated through the image cross-correlation operation (CC) based on the Fourier transform, as performed in normal PIV. This approach is known as the super-resolution PIV (see Shan and Gharib 1998). The CC estimate presented here is an adaptation of this CC operation and was developed specifically for the DDPIV technique. In this implementation, particles measured in the three-dimensional space are represented by a mathematical model and are cross-correlated in physical space. Since the particle information on position and size is necessary to operate this spatial CC, we describe briefly the DDPIV methodology hereafter.

$3 D$ particle identification and size with DDPIV. In a standard 2D imaging system, light scattered by a point source is collected through a converging lens and a single aperture, which is usually located on the lens axis. The DDPIV technique uses a mask with a multiplicity of off-axis pinhole apertures, arranged in a predefined geometrical pattern, to obtain a multiplicity of images from each scattering source. These images form the same identical geometrical pattern on the image plane, yet this pattern is scaled according to the depth location of the scattering source. For in-focus particles, 
the pattern is reduced to a point. Hence, the particle threedimensional location can be determined, through simple ray optics relationships, by measuring the centroid and size of the pattern on the image plane. The reader will find a detailed description of the DDPIV principle and assessment in the work by Pereira and Gharib (2002). Further derivations can be found in Kajitani and Dabiri (2005).

As per the particle sizing, DDPIV uses a ray optics approximation of the Mie scattering theory by which the size of the particle (either gas, liquid or solid) is inferred from its scattering intensity. A transfer function, determined through volumetric calibration, establishes the link between this intensity and a reference intensity. This latter, which characterizes the illumination conditions specific to the experimental set-up, is determined using calibrated particles of known optical and size properties. Their scattering intensity, combined with their spatial coordinates equally measured with DDPIV, constitutes the necessary basis to apply the 3D sizing procedure introduced by Pereira and Gharib (2004).

Cross-correlation estimate in DDPIV. In traditional planar digital particle image velocimetry analysis (either two or three velocity components), the pixel image cross-correlation is the de facto standard evaluation tool for estimating the particle displacement (Adrian 1991, Willert and Gharib 1991). PIV is used as the input estimate to 2D PTV in the so-called super-resolution particle image velocimetry methodology, as introduced by Keane et al (1995). For 3D situations, Pereira and Gharib (2002) describe an implementation of the crosscorrelation principle for DDPIV. In this latter approach, the displacement is estimated by performing a three-dimensional spatial cross-correlation between the particles' 3D locations. Hence, DDPIV differs from traditional PIV in that it requires first to identify all the particles in physical space, while PIV operates directly on the particle pixel images.

The DDPIV flow domain is divided into contiguous elements of volume, or 'voxels', as opposed to the pixel interrogation windows of planar PIV. A voxel is a rectangular solid, with edges parallel to the coordinate axes, and is defined by a centre and its size along each axis. Voxels are distributed on a regular lattice that encompasses the domain of interest of the flow. The flow velocity estimate is computed by crosscorrelating two time-separated subsets of particles, each subset being spatially located inside a given voxel. To perform this operation, each particle is represented by an isotropic 3D Gaussian function, thus allowing the correlation coefficient to be expressed by

$$
\begin{aligned}
R_{G_{i}, G_{j}} & =A_{i} A_{j}\left(\frac{\sqrt{2 \pi} R_{i} R_{j}}{\sqrt{R_{i}^{2}+R_{j}^{2}}}\right)^{3} \\
& \times \exp \left[-\frac{1}{2} \frac{\left(X_{c_{j}}-X_{c_{i}}-\Delta X\right)^{2}+\left(Y_{c_{j}}-Y_{c_{i}}-\Delta Y\right)^{2}}{R_{i}^{2}+R_{j}^{2}}\right. \\
& \left.-\frac{1}{2} \frac{\left(Z_{c_{j}}-Z_{c_{i}}-\Delta Z\right)^{2}}{R_{i}^{2}+R_{j}^{2}}\right]
\end{aligned}
$$

where $i$ and $j$ extend to every particle from the first and the second subsets, respectively. The amplitude $A$, the radius $R$ and the centroid $3 \mathrm{D}$ coordinates $\left(X_{c}, Y_{c}, Z_{c}\right)$ of the particle are provided by measurements using the DDPIV instrument, with
$A$ and $R$ being determined through the sizing procedure briefly outlined above. The vector $(\Delta X, \Delta Y, \Delta Z)$ that maximizes the correlation coefficient, in a mean-least-squares sense, is taken as the displacement in the corresponding voxel.

Beyond this cross-correlation process, DDPIV uses analysis principles borrowed from the 2D PIV field, but transposed to the $3 \mathrm{D}$ physical space where the interrogation unit is the voxel as opposed to the pixel window image. The following tools are applied here: voxel offset for increased velocity accuracy, voxel weighting by spatial 3D Gaussian functions for improved signal-to-noise ratio, voxel overlap for higher spatial resolution, iterative analysis for extended velocity dynamic range. The size information is also used in the spatial cross-correlation defined by equation (14) to minimize the effect of uncorrelated particles, hence contributing to a higher signal-to-noise ratio.

In order to keep the overall computation time low, the cross-correlation will be applied to a coarse grid only where the interrogation volumes are typically much larger than the neighbourhood volume. From this calculation, only a rough but robust estimate of the mean particle velocity can be expected. The interpolation of the coarse cross-correlation velocity estimates $\mathbf{u}_{\mathrm{cc}, k}$ to the convective velocity $\mathbf{u}_{\mathrm{c}, i}$ is performed via a simple Gaussian averaging,

$$
\mathbf{u}_{\mathrm{c}, i}=\frac{\sum_{k=1}^{N_{\mathrm{c}}} \gamma_{k} \cdot \mathbf{u}_{\mathrm{cc}, k}}{\sum_{k=1}^{N_{\mathrm{c}}} \gamma_{k}}
$$

where $N_{\mathrm{c}}$ is the number of coarse cross-correlation velocity estimates. The weight coefficients $\gamma_{k}$ are given by

$$
\gamma_{k}=\exp \left[-\frac{\left(\mathbf{x}_{i}-\mathbf{x}_{\mathrm{cc}, k}\right)^{2}}{H^{2}}\right], \quad k=1, \ldots, N_{\mathrm{c}}
$$

where $H$ is the characteristic length of the cross-correlation interrogation volume and $\mathbf{x}_{\mathrm{cc}, k}$ designates its $3 \mathrm{D}$ space location.

It is important to note that the above considerations are applicable to both $2 \mathrm{D}$ and $3 \mathrm{D}$ cases.

\section{Tracking schemes}

We have chosen three different two-frame particle tracking techniques, the requirements being speed of computation, reliability and simplicity. The three methods are the nearest neighbour method, a neural network method based on the work by Labonté (1999) and a relaxation method based on the work by Baek and Lee (1996).

\subsection{Nearest neighbour}

The link criterion for the nearest neighbour method is simply the minimum distance of a match,

$$
l_{i, j}=\min \left\|\mathbf{x}_{i}-\mathbf{y}_{j}\right\| \quad \text { with } \quad\left\|\mathbf{y}_{j}-\tilde{\mathbf{y}}_{i}\right\|<R_{\mathrm{s}},
$$

where $R_{\mathrm{s}}$ is the radius of the search neighbourhood as described above. Note that in equation (17) only the possible links within the search volume are considered and, therefore, a global minimum is not sought. 


\subsection{Neural network}

Our neural network approach is designed upon the two-layer network described by Labonté (1999), itself based on the selforganizing map (SOM) proposed by Kohonen (1989). The original implementation gave very bad results for our test cases. We altered the approach and used it as a first step before applying the nearest neighbour method. This procedure was used by Labonté (2000), who describes it as an enhanced version of the nearest neighbour matching algorithm. In his implementation, the neural network and the nearest neighbour schemes are operated concurrently, whereas we apply them sequentially. We shall also underline that Labonté (1999) did not assess his method against other tracking techniques and, to our knowledge, no further work has been done in this direction. Hence, this study also represents the first comparative evaluation for the neural network approach.

The neural network we are considering is composed of two similar subnetworks, each one corresponding to one of the two time steps (frames). The first network has $N$ neurons at $\mathbf{x}_{i}$ with a corresponding weight vector $\mathbf{v}_{i}$, whereas the second network has $M$ neurons at $\mathbf{y}_{j}$ with a corresponding weight vector $\mathbf{w}_{j}$. The dynamic of the network will be such that the weight vectors of the neurons will evolve towards a common vector. This self-organization is obtained via competition between the neurons of each subnetwork. In our approach, we successively present all weight vectors of the first network $\mathbf{v}_{i}$ to the weight vectors of the second network and, in an iterative process, a winner neuron $c_{i, j}$ of the second network is determined as the one with the weight vector $\mathbf{w}_{j}$ closest to $\mathbf{v}_{i}$. Then each neuron will be awarded a weight change according to

$$
\begin{aligned}
& \Delta \mathbf{v}_{i}=\alpha_{i} \cdot\left(\mathbf{w}_{\mathrm{c}_{i, j}}-\mathbf{v}_{i}\right) \\
& \Delta \mathbf{w}_{\mathrm{c}_{i, j}}=\alpha_{i} \cdot\left(\mathbf{v}_{i}-\mathbf{w}_{\mathrm{c}_{i, j}}\right)
\end{aligned} \quad \text { with } \quad j=1, \ldots, M
$$

where $\alpha_{i}$ is a scalar number between 0 and 1 , which we turn on or off according to

$$
\alpha_{i}= \begin{cases}\alpha & \text { if }\left\|\mathbf{y}_{i}-\tilde{\mathbf{y}}_{i}\right\|<\tilde{R}_{s}^{(n)} \\ 0 & \text { otherwise }\end{cases}
$$

where $\tilde{R}_{s}^{(n)}$ defines the radius of the search volume at iteration $n$. Thus, only the weights of the neurons that are within this search volume are affected. When all weight vectors have been used according to equation (18), the weights are updated to

$$
\begin{array}{ll}
\mathbf{v}_{i} \leftarrow \mathbf{v}_{i}+\Delta \mathbf{v}_{i} & i=1, \ldots, N \\
\mathbf{w}_{j} \leftarrow \mathbf{w}_{j}+\Delta \mathbf{w}_{j} & j=1, \ldots, M .
\end{array}
$$

To initialize the iterative process, the radius $\tilde{R}_{s}^{(0)}$ is set to $R_{\mathrm{S}}$ and the weight according to

$$
\mathbf{v}_{i}^{(n)}=\tilde{\mathbf{y}}_{i}, \quad \mathbf{w}_{j}^{(n)}=\mathbf{y}_{j}
$$

where $\tilde{\mathbf{y}}_{i}$ is defined by equation (13). After each iteration, $\tilde{R}_{s}$ and $\alpha$ are linearly decreased following

$$
\begin{gathered}
\tilde{R}_{s}^{(n)} \leftarrow \tilde{R}_{s}^{(n-1)}-\left(R_{\mathrm{S}}-R_{\mathrm{f}}\right) /\left(N_{\mathrm{C}}-1\right) \\
\alpha^{(n)}=R_{\mathrm{f}} / \tilde{R}_{s}^{(n)}
\end{gathered}
$$

where $R_{\mathrm{f}}$ is the final radius and $N_{\mathrm{C}}$ is the number of cycles. During the updating process, $\tilde{R}_{s}^{(n)}$ tends towards $R_{f}$ and $\alpha^{(n)}$ towards unity. After completion, a final nearest neighbour search using the updated weight vectors as a criterion is

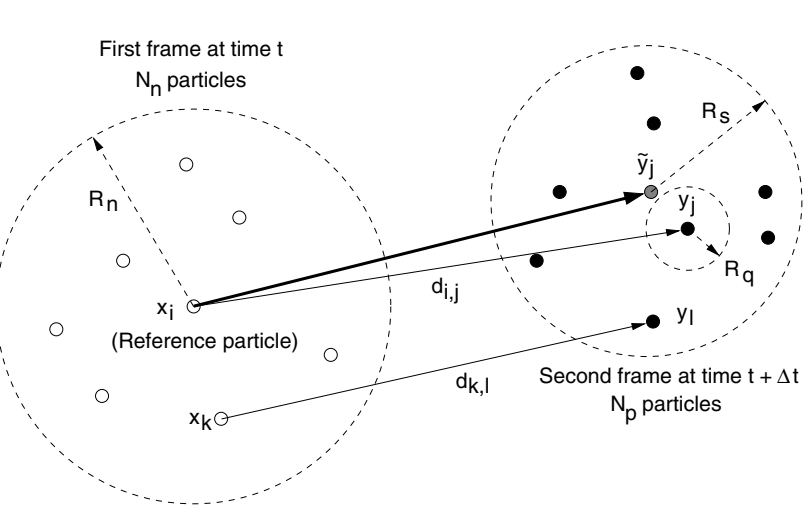

Figure 1. Relaxation principle.

performed to find the corresponding particle pairs. This neural network can be viewed as a pre-sorting routine for the nearest neighbour method, for which the weight vector of a given particle acts as an initial indicator pointing towards the matchable partner.

Labonté (2000) showed that the neural network iteratively converges towards a solution even when the number of matching particles in the initialization set (delimited by the search radius $\tilde{R}_{s}^{(0)}$ ) is very low. The algorithm was found to need only a few good matching pairs to progressively adjust itself by bringing closer the weight vectors of the matching neurons, and build a new neighbourhood with more matching particles for the next cycle.

\subsection{Relaxation method}

The relaxation method employed here is mainly the one described by Baek and Lee (1996). This is one of the first implementations of a relaxation method for particle tracking in fluid mechanics. Further developments, which are computationally more expensive, have also been published (Ohmi and Lam 1998), but have proven to yield only minor improvements. Here, we describe briefly the principle of the relaxation method with a slightly modified version of the updating process initially proposed by Barnard and Thompson (1980).

The basic concept of particle tracking in the relaxation method is to search for the most probable link of a reference particle while assuming similar displacements of its neighbour particles (the so-called quasi-rigidity condition). In an iterative process, the correct link probability is gradually increased close to unity while the other probabilities tend to zero. The scheme is described in figure 1 and can be expressed as follows. A particle $i$ in the first frame has a number of possible links $N_{\mathrm{p}}$ to the particles in the second frame satisfying

$$
\left\|\mathbf{y}_{j}-\tilde{\mathbf{y}}_{i}\right\|<R_{\mathrm{s}}
$$

where $\tilde{\mathbf{y}}_{i}$ is the estimated location of particle $i$ as per equation (13) and $R_{\mathrm{s}}$ is the search radius. At the same time, within the limit of the first frame, particle $i$ has a number of neighbour particles $N_{\mathrm{n}}$ which satisfy

$$
\left\|\mathbf{x}_{i}-\mathbf{x}_{k}\right\|<R_{\mathrm{n}}
$$

where $R_{\mathrm{n}}$ is the neighbourhood radius.

The quasi-rigidity condition, relying on a small velocity change heuristic, is applied to the neighbour particles $\mathbf{x}_{k}$ which 
show similar movement to the particle $\mathbf{x}_{i}$. Within the limits set by equations (20) and (21), we define the displacement vector $\mathbf{d}_{i, j}=\mathbf{y}_{j}-\mathbf{x}_{i}$ for a possible link from the first frame to the second one, and the neighbour displacement vector $\mathbf{d}_{k, l}=\mathbf{y}_{l}-\mathbf{x}_{k}$ of the neighbour particle $\mathbf{x}_{k}$ to its corresponding possible link $\mathbf{y}_{l}$ in the second frame. We define a weight $Q_{i, j, k, l}$ to indicate whether or not a neighbour link satisfies the quasi-rigidity condition,

$$
Q_{i, j, k, l}= \begin{cases}1 & \text { if }\left\|\mathbf{d}_{i, j}-\mathbf{d}_{k, l}\right\|<R_{\mathrm{q}} \\ 0 & \text { otherwise }\end{cases}
$$

where $R_{\mathrm{q}}$ is the quasi-rigidity radius and is typically $\approx 20 \%$ of $R_{\mathrm{s}}$. In order to make further use of the flow estimate given by equation (13), we define an additional weight $F_{i, j}$ :

$$
F_{i, j}= \begin{cases}1 & \text { if }\left\|\mathbf{d}_{i, j}-\mathbf{u}_{\mathrm{c}, i} \cdot \Delta t\right\|<R_{\mathrm{q}} \\ 0 & \text { otherwise. }\end{cases}
$$

Generally, the probability $P_{i, j}$ for particle $i$ in the first frame to match a particle $j$ in the second frame has to satisfy

$$
\sum_{j=1}^{N_{\mathrm{p}}} P_{i, j}+P_{i}^{\star}=1
$$

after each iteration, with $P_{i}^{\star}$ being the probability for particle $i$ to have no matching particle in the second frame. The initial values for $P_{i, j}$ and $P_{i}^{\star}$ can be given by

$$
P_{i, j}^{(0)}=P_{i}^{\star(0)}=\frac{1}{N_{\mathrm{p}}+1} .
$$

The match probabilities $P_{i, j}$ are updated according to the following equation,

$$
\tilde{P}_{i, j}^{(n)}=P_{i, j}^{(n-1)} \cdot\left[\begin{array}{c}
A+B \cdot\left(\sum_{k=1}^{N_{\mathrm{n}}} \sum_{l=1}^{N_{\mathrm{p}, k}} P_{k, l}^{(n-1)} \cdot Q_{(i, j, k, l)}\right) \\
+C \cdot F_{i, j}
\end{array}\right],
$$

where $N_{\mathrm{p}, k}$ is the number of possible links of a neighbour particle $k$ to the second frame. The constants are fixed as $A=0.3$ and $B=3.0$ (Barnard and Thompson 1980) and the new constant $C$ was experimentally determined as $C=1.0$.

The updated probability $\tilde{P}_{i, j}^{(n)}$ has to be normalized to make possible equation (24). This is done by using the no-match probability $P_{i}^{\star}$ of the preceding iteration:

$$
P_{i, j}^{(n)}=\frac{\tilde{P}_{i, j}^{(n)}}{\sum_{j=1}^{N_{\mathrm{n}}} \tilde{P}_{i, j}^{(n)}+P_{i}^{\star(n-1)}} .
$$

Similarly, the no-match probability is updated as follows:

$$
P_{i}^{\star(n)}=\frac{P_{i}^{\star(n-1)}}{\sum_{j=1}^{N_{\mathrm{n}}} \tilde{P}_{i, j}^{(n)}+P_{i}^{\star(n-1)}} .
$$

Ohmi and $\mathrm{Li}$ (2000) have proposed a different updating scheme for the no-match probability $P_{i}^{\star}$, which was found to yield very little improvement in our tests.

After successful termination of this iterative process, say after $N_{\mathrm{I}}$ iterations, correct matches have high probabilities and incorrect ones have low probabilities. Therefore, the most probable link $l_{i, j}$ is that with the largest match probability value.

The new constant $C$ was introduced for two reasons. First, it gives the link in the direction of the flow estimate a higher probability since it is legitimate to believe that the corresponding matching particle lies preferably in that direction. Second, it ensures a link $l_{i, j}$ even if $N_{\mathrm{n}}=0$, i.e. if a particle has no neighbours within the radius $R_{n}$. Otherwise, the no-match probability $P_{i}^{\star}$ would increase to 1 , which is obviously not correct.

Unlike the neural network, where the neighbourhood is iteratively adjusted to contain an increasingly larger number of matching particles, the relaxation algorithm works on a fixed set of particles during its iterative process. Therefore, it requires in the initial step enough matching particles to stimulate the matching process and converge towards a reliable solution. If this is not the case, the probability may not converge towards unity, in which case no matching particles are found.

\section{Test cases and discussion}

In order to test the performance of the different tracking schemes, the Burgers' vortex and a complex flow field with strong velocity gradients were chosen as the test cases. Since the test velocity flow field and, with it, the particle positions and number are known in advance, we introduce two terms to evaluate the performance of each tracking scheme: the recovery ratio $\eta_{\mathrm{r}}$ and the mismatch ratio $\eta_{\mathrm{m}}$. If $M_{\mathrm{cl}}$ is the number of links correctly identified, then $\eta_{\mathrm{r}}$ is simply defined by $M_{\mathrm{cl}}$ divided by the actual number of correct links $N_{\mathrm{cl}} \cdot \eta_{\mathrm{m}}$ is the ratio of the number of spurious (or incorrect) links $M_{\mathrm{sl}}$ divided by the total number $M_{1}$ of links found by the tracking algorithm (thus including both correct and incorrect links):

$$
\eta_{\mathrm{r}}=\frac{M_{\mathrm{cl}}}{N_{\mathrm{cl}}}, \quad \eta_{\mathrm{m}}=\frac{M_{\mathrm{sl}}}{M_{1}} .
$$

$\eta_{\mathrm{r}}$ provides a direct evaluation of the performance of the scheme, while $\eta_{\mathrm{m}}$ acts as a noise-to-signal ratio and indicates whether or not the algorithm can reliably discriminate and dismiss unpaired and/or noise particles.

DDPIV is our 3D measurement technique of choice for implementing the tracking schemes introduced in the previous section. To evaluate the performance of these algorithms, synthetic 3D particle fields are created and processed to obtain the DDPIV cross-correlation-based estimates of the velocity fields, as explained in section 2.4.

\subsection{Burgers' vortex flow case}

The Burgers' vortex is a steady solution of the Navier-Stokes equation where the action of strain and viscosity balance each other to give a vortex core of steady finite size. The Burgers' vortex is a laminar but complex structure and displays a range of scales defined by the distance between successive windings of its streamlines. The equation of Burgers' vortex is given in Cartesian coordinates as

$$
V=\frac{\Gamma}{2 \pi r} \cdot\left[1-\exp \left(-\sigma \frac{r^{2}}{4 v}\right)\right] \quad \text { with } \quad r=\sqrt{x^{2}+y^{2}}
$$

where $\sigma$ is the rate-of-strain, $\Gamma$ is the circulation and $v$ is the kinematic viscosity. For our test, we also impose a linear 


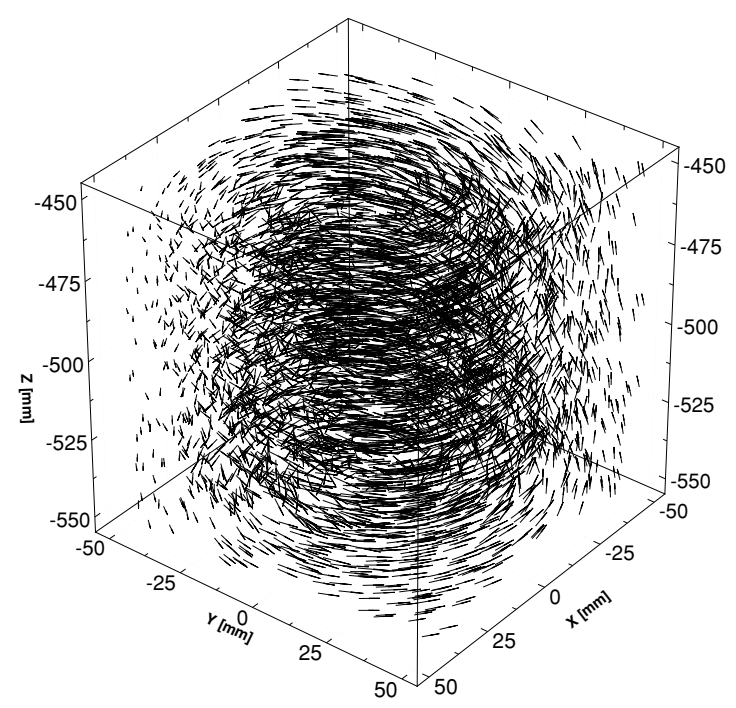

Figure 2. Burgers' vortex flow case: synthetic 3D vector field with 5000 vectors; $\Gamma=200 \mathrm{~mm}^{2} \mathrm{~s}^{-1}, \sigma=0.01 \mathrm{~s}^{-1}, v=1 \mathrm{~mm}^{2} \mathrm{~s}^{-1}$.

Table 1. Tracking parameter $\Phi$ and displacement $|\mathbf{x}|_{\max }$ for 12 values of $\Delta t ; d_{0} \approx 3.628 \mathrm{~mm}$.

\begin{tabular}{ccl}
\hline$\Delta t(\mathrm{~s})$ & $|\mathbf{x}|_{\max }(\mathrm{mm})$ & $\Phi$ \\
\hline 0.5 & 0.513 & 7.069 \\
1.0 & 1.026 & 3.535 \\
1.5 & 1.539 & 2.356 \\
2.0 & 2.053 & 1.767 \\
2.5 & 2.566 & 1.414 \\
3.0 & 3.079 & 1.178 \\
3.5 & 3.592 & 1.009 \\
4.0 & 4.105 & 0.883 \\
4.5 & 4.618 & 0.785 \\
5.0 & 5.132 & 0.707 \\
7.5 & 7.697 & 0.471 \\
10 & 10.263 & 0.353 \\
\hline
\end{tabular}

velocity gradient $\delta=0.001 \mathrm{~s}^{-1}$ normal to the $X-Y$ plane to simulate three-dimensionality.

The measurement domain is a cubic volume $V_{0}$ of $100 \times$ $100 \times 100 \mathrm{~mm}^{3}$ with $X \in[-50,50] \mathrm{mm}, Y \in[-50,50] \mathrm{mm}$ and $Z \in[-450,-550] \mathrm{mm}$. The parameters of the Burgers' vortex are fixed as follows: $\Gamma=200 \mathrm{~mm}^{2} \mathrm{~s}^{-1}, \sigma=0.01 \mathrm{~s}^{-1}$ and $v=1 \mathrm{~mm}^{2} \mathrm{~s}^{-1}$.

Tracking performance. Data sets with 5000 particles/vectors normally distributed in space are synthetically generated. The tracking parameter $\Phi$ is varied by choosing different $\Delta t$, thus varying the maximum particle displacement $|\mathbf{x}|_{\max }$, as reported in table 1 . The initial number $N_{0}$ of particles in the measurement volume is unchanged for each case, hence $d_{0}$ is constant with $d_{0} \approx 3.628 \mathrm{~mm}$. To simulate the practical situation where particle detection fails, the particle population is randomly reduced by $5 \%$ between two consecutive frames, thus creating unpaired particles. A sample snapshot of the synthetic vector field is shown in figure 2.

The search radius $R_{\mathrm{s}}$ was set constant to $5 \mathrm{~mm}$ for the nearest neighbour and the neural network methods, except for $\Delta t>5 \mathrm{~s}$ where $R_{\mathrm{s}}=10 \mathrm{~mm}$. The final radius $R_{\mathrm{f}}$ of the neural network was equal to $|\mathbf{x}|_{\max }+5 \%$. For the relaxation

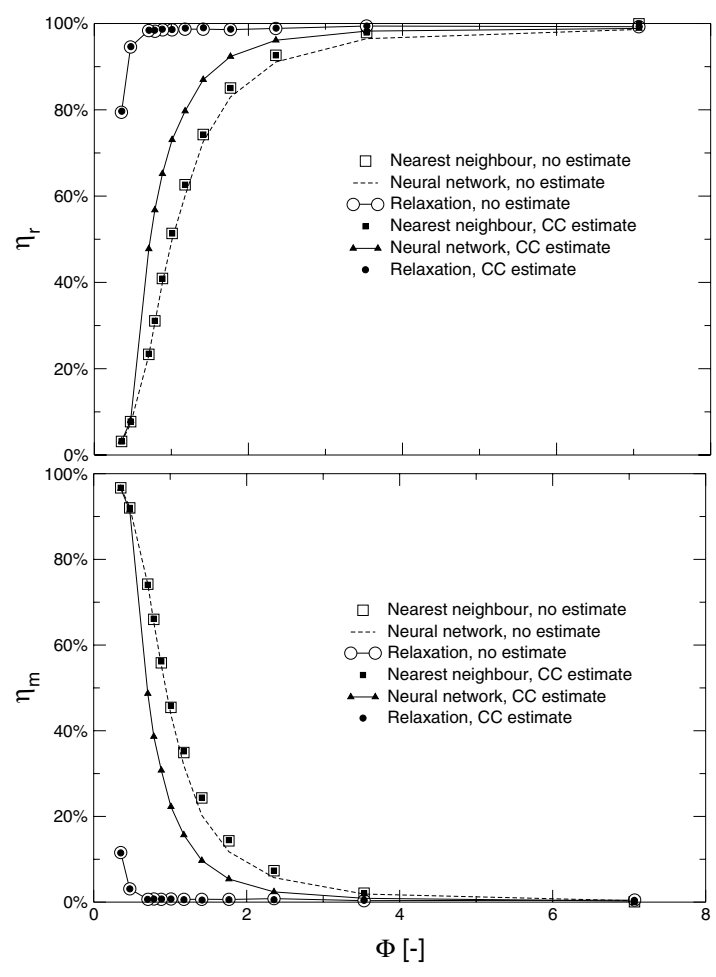

Figure 3. Burgers' vortex flow case: recovery ratio $\eta_{\mathrm{r}}$ and mismatch ratio $\eta_{\mathrm{m}}$ versus the tracking parameter $\Phi$, with and without cross-correlation (CC) velocity estimate.

method, $R_{\mathrm{s}}$ was varied between 5 and $6 \mathrm{~mm}$ for $\Delta t \leqslant 5 \mathrm{~s}$, and between 8 and $10 \mathrm{~mm}$ otherwise. The neighbourhood radius $R_{\mathrm{n}}$ was equal to $100 \% \cdot R_{\mathrm{S}}$ and the quasi-rigidity radius $R_{\mathrm{q}}$ was $20 \% \cdot R_{\mathrm{s}}$. The number of iterations $N_{\mathrm{I}}$ for the relaxation method and the number of cycles $N_{\mathrm{C}}$ for the neural network method were both set to 20 .

The calculation of the mean flow velocity estimate using the cross-correlation analysis was performed with $10 \times 10 \times$ $10 \mathrm{~mm}^{3}$ cubic interrogation domains (or voxels), resulting in a field with $11 \times 11 \times 11=1331$ velocity vectors as the flow estimate for the tracking schemes. Figure 3 shows the recovery ratio $\eta_{\mathrm{r}}$ and the mismatch ratio $\eta_{\mathrm{m}}$ as a function of the tracking parameter $\Phi$ for the three tracking methods, with and without flow velocity estimate. It is seen that all methods perform very well for $\Phi>3.5$, with a recovery ratio $\eta_{\mathrm{r}}$ larger than $97 \%$. For lower $\Phi$, the nearest neighbour and the neural network methods perform equally, with a slight advantage for the nearest neighbour approach. Yet, both perform very poorly compared to the relaxation method. This latter scheme maintains a high yield of more than $98 \%$ for values of $\Phi>0.7$, while the other two schemes demonstrate a regular and pronounced decrease in efficiency for $\Phi<3.5$. This performance loss is due to the fact that the corresponding maximum particle displacement $|\mathbf{x}|_{\max }$ is larger than the particle mean distance $d_{0}(\approx 3.628 \mathrm{~mm})$, see table 1 . The nearest neighbour scheme is obviously particularly affected for it works best, by concept, when the displacements are small with respect to the particle mean distance. This also affects directly the results of the neural network, since we use the nearest neighbour scheme as its final tracking stage. 
The mean flow estimate calculated using the DDPIV cross-correlation analysis clearly improves the performance of the neural network scheme with a recovery yield enhanced by as much as $26 \%$ for $\Phi=0.785$. The flow estimate has an imperceptible $(\ll 1 \%)$ effect on the performance of the nearest neighbour and of the relaxation methods. As per the mismatch ratio $\eta_{\mathrm{m}}$, the relaxation method again gives the best results, with less than $1 \%$ of spurious links for $\Phi>0.7$. The neural network, when supported by the CC estimate, displays a higher level of confidence than the nearest neighbour scheme, indicating that it more efficiently avoids the unpaired particles and generates fewer spurious links.

To get a qualitative picture of the performance of the tracking techniques tested, we show in figure 4 a projection on the $X-Y$ plane of the resulting vector field for the case $\Phi=0.707\left(|\mathbf{x}|_{\max }=5.132 \mathrm{~mm}\right)$, using for each scheme the local velocity cross-correlation estimate. The figures clearly demonstrate the superior performance of the relaxation method, which finds more than $98.5 \%$ of the actual exact vector field. The results for the neural and the nearest methods are, respectively, $47.8 \%$ and $23.5 \%$. Each method also finds a number of spurious vectors, which are the consequence of wrong matching of particles. For the case illustrated in figure 4 , we have $\eta_{\mathrm{m}}=0.7 \%$ for the relaxation method, $48.7 \%$ for the neural scheme and $74.1 \%$ for the nearest algorithm.

Time performance. In addition to the tracking efficiency, it is of practical interest to evaluate the speed performance of a given tracking scheme. We choose to perform this study on the same Burgers' vortex flow with a maximum displacement $|\mathbf{x}|_{\max }$ set constant to $0.513 \mathrm{~mm}$. The number of particles is increased from 1000 to 50000 , for a corresponding $\Phi \in[3.28,12.09]$. In this range, the three algorithms perform similarly in terms of tracking, as shown in figure 3, with a yield of correct found tracks higher than $97 \%$ and a very low $\eta_{\mathrm{m}}$. No particles are removed from the field between the two frames and the algorithms are applied without velocity estimate. The search radius $R_{\mathrm{S}}$ was set to $5 \mathrm{~mm}$ for all the schemes, the final radius $R_{\mathrm{f}}$ of the neural network was equal to $2 \mathrm{~mm}$, and the other parameters remained unchanged with respect to the previous analysis. Figure 5 represents the processing time $t^{*}$ as a function of the number of particles $N_{0}$, with $t^{*}$ being the actual time normalized by the time required by the nearest neighbour method for $N_{0}=50000(t=44.485 \mathrm{~s})$. We find that this time is closely proportional to $N_{0}^{2}$ (correlation coefficient $\approx 1$ ), as shown by the power- 2 law fit curves reported in the figure.

The relaxation scheme requires processing times that are less than twice as long as those of the nearest neighbour approach. In contrast, the neural network scheme displays the longest processing times of all tracking methods. This time is 10 to 12 times longer than that of the relaxation method, and 20 to 25 times longer than the nearest neighbour method. Moreover, no cross-correlation estimate has been calculated, hence the tracking performance of the neural network analysis is equivalent to that of the nearest neighbour, as shown in the previous section. It is important to note that the neural network scheme has often been praised in the past (Labonté 1999) for its being potentially well suited for parallel computation. Indeed, all the weight increments can be calculated separately and at the same time, and all the neurons can consequently have their
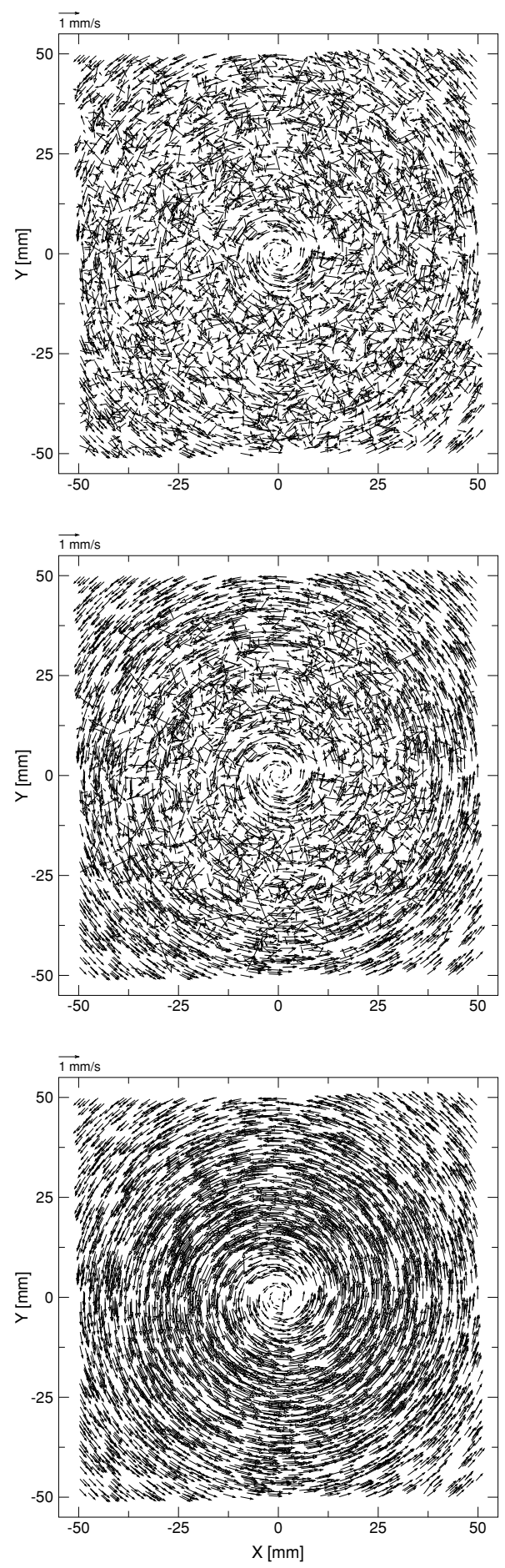

Figure 4. Burgers' vortex flow case: $X-Y$ projection of the calculated velocity vector field using three tracking schemes with cross-correlation estimate. Top: nearest neighbour; centre: neural network; bottom: relaxation. $\Phi=0.707$.

weights updated simultaneously. In a parallel computation, the time required for this updating cycle would therefore be independent of the number of neurons. However, the two other techniques would also benefit from such a parallel implementation, since the analysis could be equally shared among different processing units, and the claimed advantage of the neural network approach would undoubtedly vanish. 


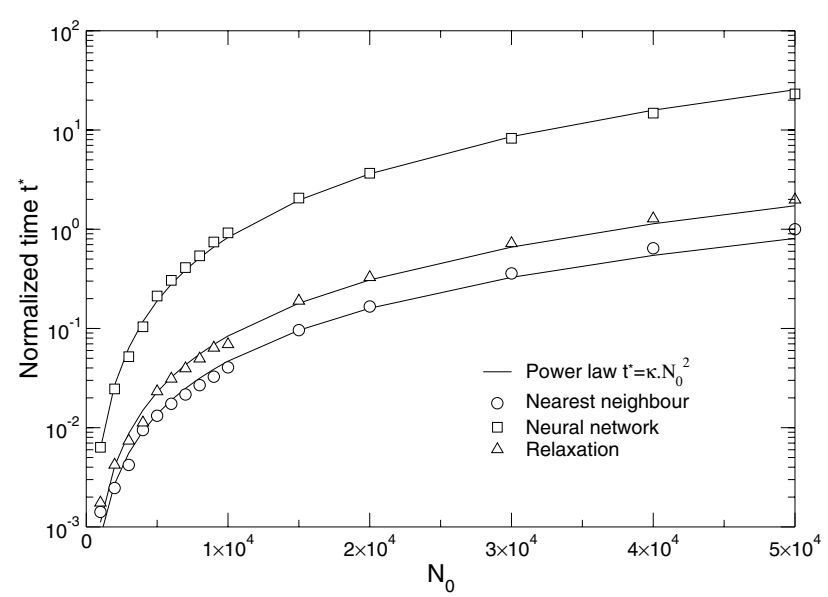

Figure 5. Processing time for the three tracking schemes versus the number of particles; $|\mathbf{x}|_{\max }=0.513 \mathrm{~mm}$, no velocity estimate.

\subsection{High density flow case}

PTV is commonly believed to be limited to flows lightly seeded, hence to low density particle images. The goal of this section is to demonstrate the applicability of tracking to relatively high density $3 \mathrm{D}$ particle fields with strong velocity gradients. The synthetic flow considered here is the result of the contribution of 1000 Burgers' vortices randomly distributed in the same cubic domain as in the previous case. The parameters of the Burgers' vortices are fixed as follows: $\Gamma=2.5 \mathrm{~mm}^{2} \mathrm{~s}^{-1}, \sigma=1 \mathrm{~s}^{-1}$ and $v=1 \mathrm{~mm}^{2} \mathrm{~s}^{-1}$. The rate-of-strain and the circulation are randomized to simulate multi-scale vortical structures. In addition, the circulation is randomly positive or negative. The linear velocity gradient along the $Z$-axis is $\delta=0.001 \mathrm{~s}^{-1}$.

Thus, the resulting velocity field is the time-evolving contribution of a family of random Burgers' vortex structures. At each step in time, every vortex centre is convected according to the local contribution as determined at the previous time step, and a new velocity field is calculated based on the updated locations of the vortices. The flow is seeded with 20000 particles, the positions and velocity of which are known. A sample snapshot of the flow is shown in figure 6.

The tracking parameter $\Phi$ is varied between 0.245 and 3.658 by changing the interframe time $\Delta \mathrm{t}$. The search radius $R_{\mathrm{S}}$ was set constant to $2.5 \mathrm{~mm}$ for the nearest neighbour and the neural network methods. The final radius $R_{\mathrm{f}}$ of the neural network was equal to $|\mathbf{x}|_{\max }+5 \%$. For the relaxation method, $R_{\mathrm{S}}$ was varied between 2.5 and $5 \mathrm{~mm}$. The remaining parameters (the neighbourhood radius $R_{\mathrm{n}}$ and the quasi-rigidity radius $R_{\mathrm{q}}$ ) remained unchanged with respect to the previous analysis. The mean flow velocity estimate using the cross-correlation analysis was calculated based on $5 \times 5 \times 5 \mathrm{~mm}^{3}$ voxels, resulting in a field with $21 \times 21 \times 21=9261$ velocity estimates.

Figure 7 shows the recovery ratio $\eta_{\mathrm{r}}$ and the mismatch ratio $\eta_{\mathrm{m}}$ as a function of the tracking parameter $\Phi$ for the three tracking methods, with and without velocity estimate. All methods are found to perform very well for $\Phi>2$, with a recovery ratio $\eta_{\mathrm{r}}$ larger than $97 \%$. Below this value, the relaxation algorithm maintains a high tracking yield for $\Phi>0.717$, while the other two schemes experience a strong

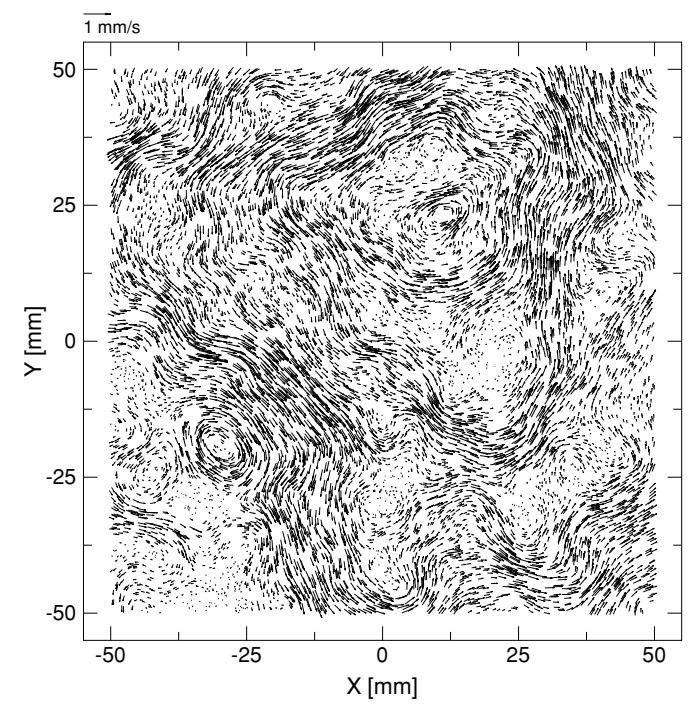

Figure 6. High density flow case: $X-Y$ projection of the velocity vector field (one vector out of every two is represented).

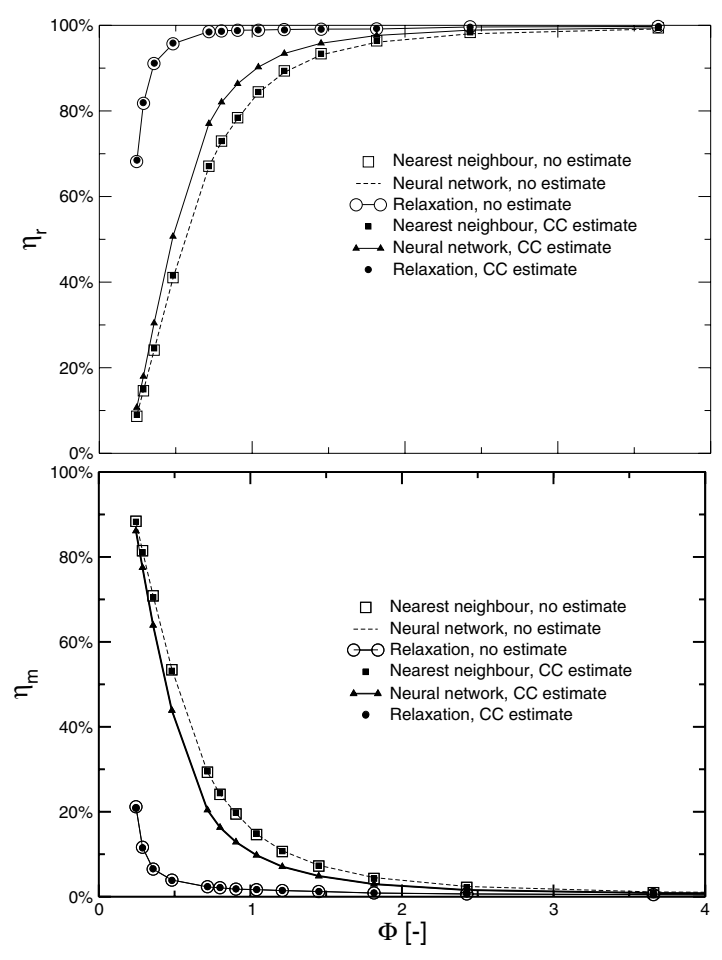

Figure 7. High density flow case: recovery ratio $\eta_{\mathrm{r}}$ and mismatch ratio $\eta_{\mathrm{m}}$ versus the tracking parameter $\Phi$, with and without cross-correlation (CC) velocity estimate.

decay in efficiency. The cross-correlation estimate brings little improvement to the performance of the tracking methods except for the neural network scheme, the tracking efficiency of which is enhanced by $10 \%$ for $\Phi=0.717$. Interestingly, the relaxation method behaves almost exactly as in the previous flow case, while the two concurrent schemes show different results. As regards the mismatch ratio $\eta_{\mathrm{m}}$, the neural network with the CC velocity estimate performs better than the nearest neighbour, this latter displaying the highest level of noise links of the three methods. 


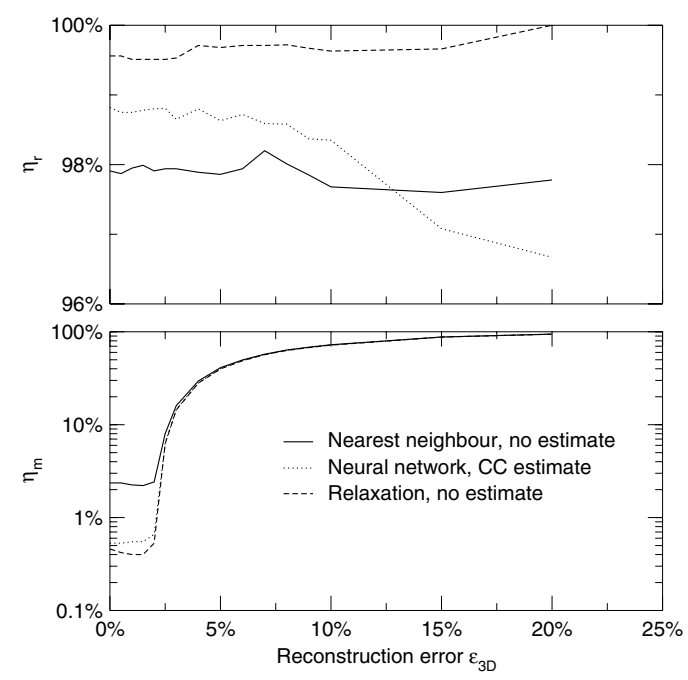

Figure 8. Effect of reconstruction errors on the recovery ratio $\eta_{\mathrm{r}}$ and the mismatch ratio $\eta_{\mathrm{m}}$.

\subsection{Effect of DDPIV reconstruction errors}

DDPIV is subject to errors in the three-dimensional reconstruction of the particles' positions. Pereira and Gharib (2002) established the link between these errors and the optical and geometrical parameters that define a DDPIV system. They showed that the uncertainty on the Z-location was quadratically dependent upon $Z$, while that relative to the inplane components $X$ and $Y$ would vary linearly with $Z$. In order to investigate the effect of this source of error on the tracking performance of the three algorithms under consideration here, we use the single vortex flow case above and introduce a random error $\varepsilon_{3 \mathrm{D}}$ in the particle spatial location. The case $\Phi=3.535\left(|\mathbf{x}|_{\max }=1.026 \mathrm{~mm}\right)$ is selected as the three schemes give similar results for an error-free system, see figure 3 , with a very high recovery ratio $\left(\eta_{\mathrm{r}}>97 \%\right)$ and a low mismatch ratio $\left(\eta_{\mathrm{m}}<2 \%\right)$. $\varepsilon_{3 \mathrm{D}}$ is defined as a percentage of the maximum displacement $|\mathbf{x}|_{\max }$. Note that, for a given $\varepsilon_{3 \mathrm{D}}$, the applied error is randomly distributed in the interval $\left[-\varepsilon_{3 \mathrm{D}} / 2,+\varepsilon_{3 \mathrm{D}} / 2\right]$ on each spatial direction. As per the analysis found in Pereira and Gharib (2002), the error in the $Z$-location is forced to be five times higher than that in $X$ and $Y$.

Figure 8 represents the recovery and mismatch ratios as a function of $\varepsilon_{3 \mathrm{D}}$. The recovery ratio is higher than $97 \%$ and almost constant over the range of location errors considered here, except for the neural network. The performance of this latter scheme remains at an intermediate level between that of the nearest neighbour (average 97.8\%) and that of the relaxation schemes (average $99.6 \%$ ) for errors $\varepsilon_{3 \mathrm{D}}$ lower than $10 \%$. For higher reconstruction errors, the efficiency of the neural network progressively weakens, reaching levels inferior to those of the nearest neighbour algorithm. In terms of mismatch ratio $\eta_{\mathrm{m}}$, the three schemes behave similarly as expected for $\Phi=3.535$ (see figure 3), with a slightly better result for the relaxation algorithm. Although the recovery ratio is very high, the actual number of links correctly identified progressively decreases. Indeed, the increasing value of $\eta_{\mathrm{m}}$ for $\varepsilon_{3 \mathrm{D}}>2 \%$ indicates a growing number of spurious vectors being identified. The steep increase observed at $\varepsilon_{3 \mathrm{D}}=2 \%$

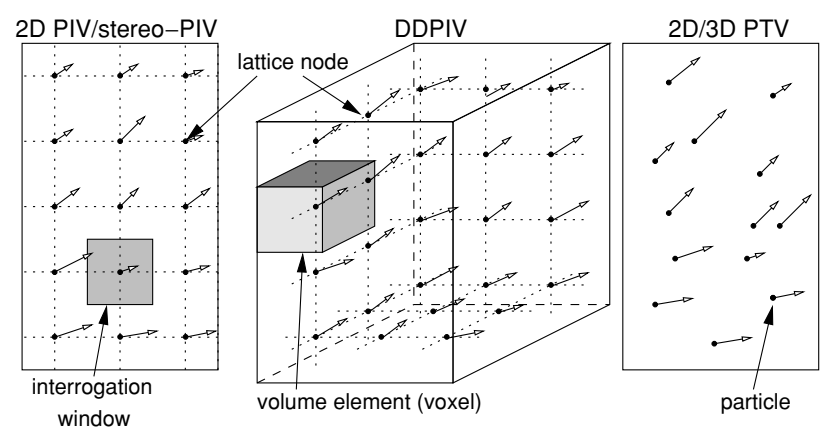

Figure 9. Lattice layout for planar PIV and DDPIV versus PTV.

corresponds to a transition point where the error in $Z$ reaches approximately $10 \%$ of the maximum displacement $|\mathbf{x}|_{\max }$ (i.e. $\approx 0.1 \mathrm{~mm}$ ), which is also close to the actual minimum displacement in the generated particle field considered in this analysis $(0.094 \mathrm{~mm})$. Since the location error $\varepsilon_{3 \mathrm{D}}$ is related in absolute terms to the maximum displacement, the local relative error can be very large (i.e. $>100 \%$ ) for small displacements, particularly in the central region of the vortex where the velocity tends to zero. This translates into new spurious links and added RMS error as $\varepsilon_{3 \mathrm{D}}$ increases.

\subsection{Tracking versus DDPIV cross-correlation}

Our intent in this section is to compare the velocity field calculated by the tracking method with that obtained using the DDPIV spatial cross-correlation (CC). An immediate comparison between the two fields is not possible for the PTV vector field is linked to the particle position, hence it is randomly distributed in space, while the PIV vector field is attached to a lattice of points. Planar PIV (standard and stereoscopic) and DDPIV both map the vector field onto a grid of regularly spaced points, each of which corresponds to the centre of an interrogation domain: a rectangle for PIV and a rectangular parallelepipedic volume element-or voxel-for DDPIV, see figure 9.

To allow the comparison with the DDPIV vector field, the voxel inside which we perform the spatial cross-correlation (see equation (14)) is centred on the particle itself and not on the node of a regular lattice. Thus, we also obtain a spatially randomly distributed set of $\mathrm{CC}$-determined vectors positioned on exactly the same locations as the PTV vectors. This approach has the main advantage of allowing one to compare fairly the two calculations, since the result is not biased by any sort of interpolation or average that would be otherwise required between the random PTV and the organized DDPIV vector fields. The DDPIV cross-correlation is performed in volume elements with an initial size of $10 \times 10 \times 10 \mathrm{~mm}^{3}$. We allow this interrogation volume to have a variable size in order to reach a minimum number of seven particles per voxel, as recommended by Pereira and Gharib (2002). The analysis is performed using the processing tools outlined in section 2.4, such as the voxel offset technique.

We represent in figure 10 the root mean square (RMS) error as a function of the reconstruction error $\varepsilon_{3 \mathrm{D}}$. The RMS is defined as the square root of the mean sum of squares of the difference vector $\left(\varepsilon_{X}, \varepsilon_{Y}, \varepsilon_{Z}\right)$ between the PTV/CC velocity vector and the theoretical quantity given by equation (30). We 


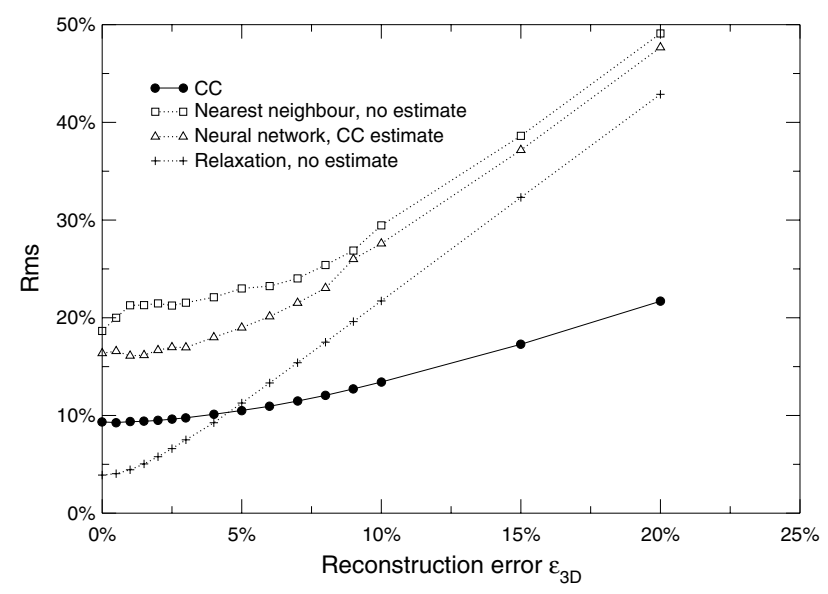

Figure 10. Tracking and DDPIV cross-correlation RMS errors versus the reconstruction error $\varepsilon_{3 \mathrm{D}}$.

display the RMS error as a percentage of the maximum velocity given by $|\mathbf{x}|_{\max }=1.026 \mathrm{~mm}$. The plot gives rise to several important comments. The relaxation algorithm proves to be superior to the neural network and nearest neighbour schemes, with the latter being the most perturbed by the reconstruction error. The PTV method, schemewise, is much more sensitive to the reconstruction error than the cross-correlation approach. The RMS error associated with the PTV analysis increases steeply to levels as high as $50 \%$, with a sensibly linear trend common to all three schemes for $\varepsilon_{3 \mathrm{D}}>10 \%$. In contrast, the RMS error obtained with the CC method increases smoothly and monotonically from $10 \%$ to $20 \%$. This result was expected since the CC method is in essence a statistical approach that makes use of the complete particle information available within the interrogation domain to build a mean estimate of the velocity therein. In this sense, the $\mathrm{CC}$ technique acts as a low-pass filter that removes the spatial fluctuations of smaller wavelength, such as those introduced by the particle reconstruction. Hence, it is naturally less prone to random errors. The relatively high error $(10 \%)$ obtained for $\varepsilon_{3 \mathrm{D}}=0$ derives from this smoothing effect, especially present in the core of the vortex where the curvature of the flow streamlines is high. On the other hand, PTV considers the single particle information and is therefore directly affected by the local error. Yet, the relaxation scheme is seen to perform even better than the $\mathrm{CC}$ for $\varepsilon_{3 \mathrm{D}}<5 \%$. As expected, the neural network algorithm performs better than the nearest neighbour scheme, for it is employed as a pre-processor to purposely enhance the nearest neighbour particle matching.

Although the PTV schemes lead in general to higher RMS errors, it must be pointed out that this error is in its totality due to the incorrectly identified particle pairs. Figure 11 represents the distribution of the error vector $\left(\varepsilon_{X}, \varepsilon_{Y}, \varepsilon_{Z}\right)$ for the PTV analysis, all three schemes combined, against that obtained with the DDPIV cross-correlation analysis for three levels of the location error $\varepsilon_{3 \mathrm{D}}(0,5$ and $10 \%)$. The PTV error distributions match the actual range of $\varepsilon_{3 \mathrm{D}}$ very well. In particular, the $4 \%$ RMS error found for $\varepsilon_{3 \mathrm{D}}=0$ for the relaxation algorithm, see figure 10 , is actually caused by only very few wrong links $(0.46 \%)$, as one can see from the plot of the mismatch ratio $\eta_{\mathrm{m}}$ in figure 8 . A similar observation can be made for the other two schemes. For

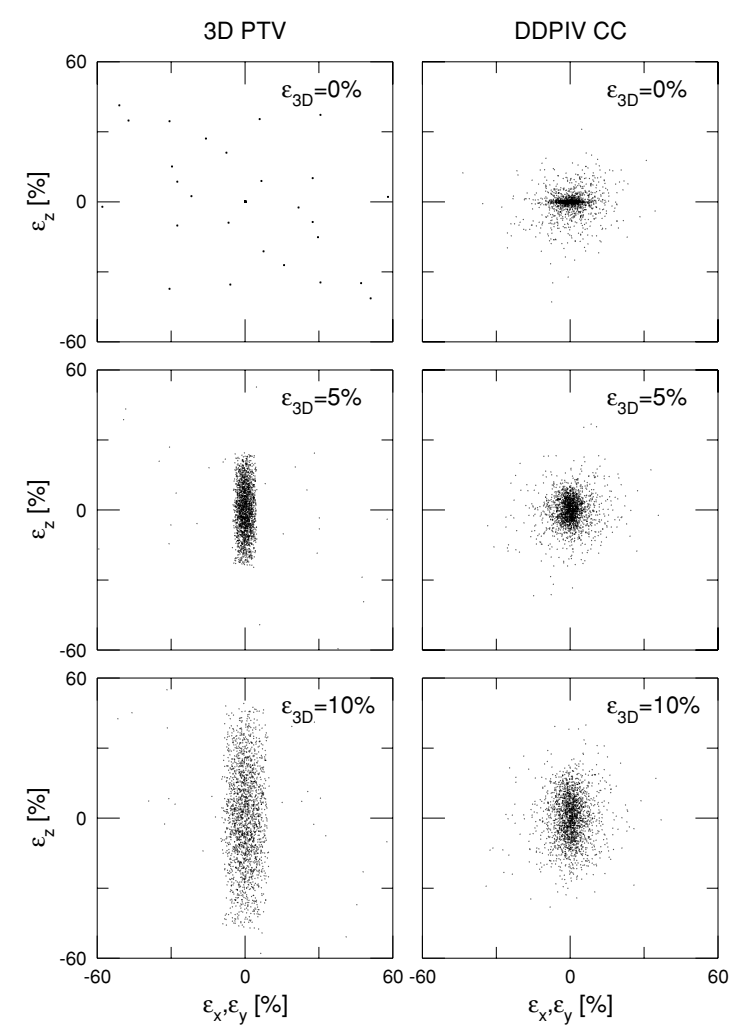

Figure 11. Error distribution for 3D PTV (nearest neighbour, neural network and relaxation schemes) and DDPIV cross-correlation, for $\varepsilon_{3 \mathrm{D}}=0,5$ and $10 \%$.

$\varepsilon_{3 \mathrm{D}}=5$ and $10 \%$, the error remains bounded by the lower and upper limits of the actual error, with the $Z$-error $\varepsilon_{Z}$ being 5 times larger than the in-plane components $\varepsilon_{X}$ and $\varepsilon_{Y}$. In contrast to the tracking approach, the DDPIV spatial crosscorrelation remains little affected by the change of $\varepsilon_{3 \mathrm{D}}$, as already commented upon (see figure 10). The error is normally distributed around 0 and ranges from -20 to $+20 \%$. The higher error in $Z$ becomes visible only for $\varepsilon_{3 \mathrm{D}}>5 \%$, with a higher dispersion observed along the $Z$-axis.

These results confirm the intuitive and well-corroborated fact that the tracking methods are more sensitive to the measurement errors than a CC-based approach. Yet, the use of an adequate tracking scheme such as the relaxation algorithm allows a more accurate reconstruction of the actual velocity field, especially when the errors of the measurement system are small, thus adding to the higher spatial resolution intrinsic to the PTV method. Furthermore, it is important to stress the fact that the error levels registered for the PTV schemes are, in particular for reduced particle location errors $\left(\varepsilon_{3 \mathrm{D}}<5 \%\right)$, largely caused by few and sparse spurious links. The resulting erroneous vectors can be easily removed through an outlier correction procedure. Instead, the errors related to the $\mathrm{CC}$ analysis extend to every interrogation cell and derive from the averaging process proper to the $\mathrm{CC}$ operation. In this case, smaller interrogation domains can reduce the error, but a compromise has to be settled with respect to the particle density required for a correct CC calculation. Such a requirement is not needed for PTV. 


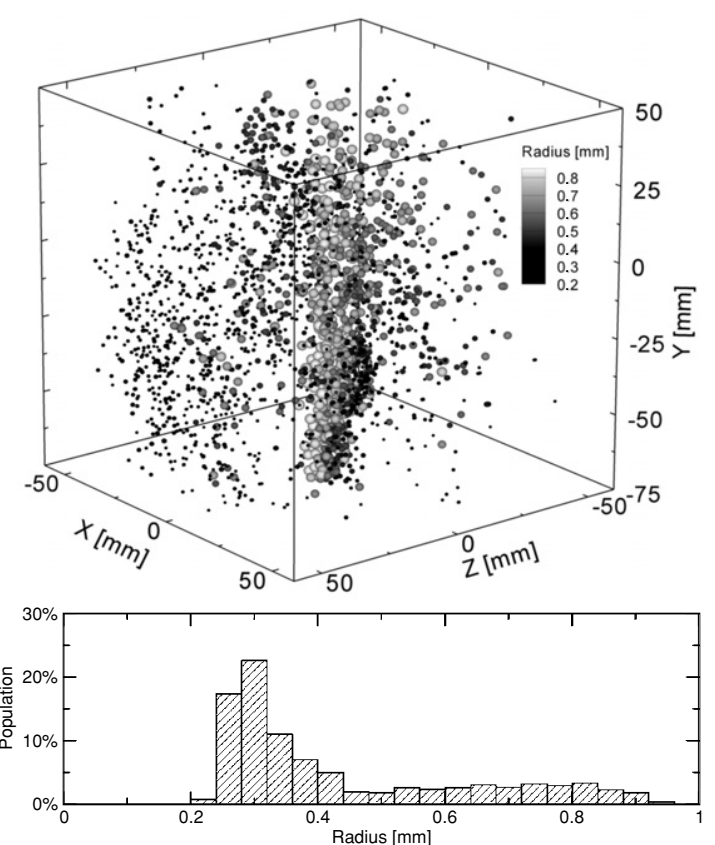

Figure 12. Snapshot of the measured 3D bubble/particle field and corresponding size distribution.

\section{Sample application: bubble plume}

The tracking algorithms presented here have been implemented as part of a current effort to address the question of the collective dynamics of multi-bubble systems. A model for bubble-bubble interaction, as yet under development, has been devised to describe the dynamical behaviour of a population of bubbles. To validate this model, Lagrangian tracking was essential, hence PTV was preferred over PIV (as implemented in DDPIV through spatial cross-correlation). We designed a bubble plume experiment, where density and size of bubbles could be adjusted. The data presented here, which include location and size of bubbles, are part of the information gathered during this effort.

An air bubble generator is placed at the bottom of a $200 \times$ $200 \times 300 \mathrm{~mm}^{3}$ water tank, and creates a continuous stream of rising sub-millimetre air bubbles. The water is also seeded with solid polymer particles with a density of $1.05 \mathrm{mg} \mathrm{mm}^{-3}$ and a size distribution with a mean value at $300 \mu \mathrm{m}$. To compensate for the slight difference in density, $21 \%$ glycerin is added in weight to the water. $1024 \times 1024$ pixels images are recorded with the DDPIV instrument at a frequency of $30 \mathrm{~Hz}$. The measurement domain is $100 \times 100 \times 150 \mathrm{~mm}^{3}$ and is illuminated by a stroboscopic light source, duly synchronized with the image acquisition process. The light $3 \mathrm{D}$ pattern is calibrated to allow the particle sizing according to Pereira and Gharib (2004). Because DDPIV cannot separate the two species simultaneously present in the fluid, the solid particle field is measured before initiating the bubble plume. Hence, by tracking the solid particles in time, one can isolate the bubbly phase. The detailed description of the long-time tracking of particles is beyond the scope of the present work.

Each flow snapshot contains an average of 3000 bubbles/particles. Since DDPIV provides the 3D coordinates and size (see section 2.4) of every single scattering source, it is possible to reconstruct and visualize the three-dimensional

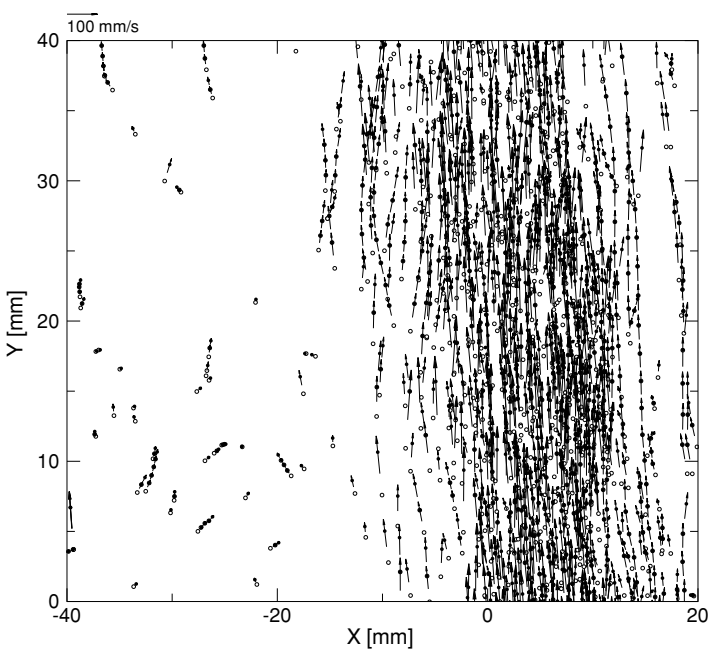

Figure 13. PTV vector field projected on the $X-Y$ plane, with first (०) and second (•) particle fields; $Z \in[0,5] \mathrm{mm}$.

particle field. A sample reconstructed field is shown in figure 12. One can easily identify the bubble plume in the central part of the observation domain. The size distribution reported in this figure clearly shows two separate but overlapping distributions, with a peak at $300 \mu \mathrm{m}$ corresponding to the mean size of the polymer particles.

We apply the relaxation algorithm without velocity estimate. Figure 13 shows a sequence of PTV vector fields in a section of the bubble plume $(Z \in[0,5] \mathrm{mm})$ and projected on the $X-Y$ plane for clarity of display. The vectors are positioned, as per equation (2), at the centre point of the corresponding particle/bubble pair, which is also reported in figure 13 with the symbols $\circ$ and - representing the particle/bubble, respectively, in the first and second frames. The solid particles in the bulk of the fluid are visible on the left side of the figure, slowly entrained by the high speed jet created by the rising bubbles.

\section{Concluding remarks}

Three algorithms have been implemented for general 3D particle tracking velocimetry (PTV): the nearest neighbour approach, the neural network and the relaxation method. These schemes have been evaluated by means of the defocusing digital particle image velocimetry (DDPIV) technique. The performance of the tracking schemes has been established based on synthetic volumetric information.

Generally, it can be stated that the relaxation scheme is the method of choice, since its performance is superior for low and high tracking parameter $\Phi$. Moreover, this technique was found to be very robust and little sensitive to the tracking parameters, unlike the other two schemes. When operated without a mean velocity estimate, the neural network, used here to enhance a nearest neighbour particle matching algorithm, yields little or no improvement over the sole nearest neighbour scheme. Furthermore, these two schemes yield overall much poorer results for low $\Phi$ than the relaxation method. In terms of processing time, the nearest neighbour method was found to be the fastest technique, while the neural network algorithm was 20 to 25 times slower and the relaxation scheme only twice as slow. 
The potential benefit of using a mean flow estimate, calculated here with the DDPIV spatial cross-correlation (CC), has also been evaluated. It has shown little or no effect on the performance of the tracking schemes, except for the neural network method for which a noticeable gain has been observed, at the expense of an increased processing time. A comparison, in terms of RMS errors, of the different tracking schemes against the DDPIV cross-correlation analysis showed that PTV is more sensitive to the DDPIV particle reconstruction errors than the correlation approach. The relaxation scheme was shown to produce lower RMS errors than the concurrent schemes. For reduced measurement errors, the relaxation algorithm could yield even lower RMS errors than the CC method, thus contributing to higher accuracy and adding to the intrinsically greater spatial resolution of PTV. To perform correctly, the relaxation method requires a number of matching particles as a stimulus. If this is not the case, the neural network approach, by way of its adaptive nature, is better suited.

Future work will address the long-time trajectory reconstruction from two-frame tracking data, as well as spurious track filtering. With the advent of a new generation of tracking algorithms (variational approach, volume deformation), we also hope to increment the tracking capability of DDPIV. In that view, one line of development will investigate the possibility of using PTV as an enhancement tool for the spatial particle cross-correlation unique to DDPIV.

\section{References}

Adrian R J 1991 Particle-imaging techniques for experimental fluid mechanics Ann. Rev. Fluid Mech. 23 261-304

Baek S J and Lee S J 1996 A new two-frame particle tracking algorithm using match probability Exp. Fluids 22 23-32

Barnard S T and Thompson W B 1980 Disparity analysis of images IEEE Trans. Pattern Anal. Mach. Intell. 2 333-40

Barnhart D H, Adrian R J and Papen G C 1994 Phase-conjugate holographic system for high-resolution particle image velocimetry Appl. Opt. 33 7159-70

Grant I and Pan X 1995 An investigation of the performance of multi-layer neural networks applied to the analysis of PIV images Exp. Fluids 19 159-66

Kajitani L and Dabiri D 2005 A full three-dimensional characterization of defocusing digital particle image velocimetry Meas. Sci. Technol. 16 790-804
Kasagi N and Nishino K 1991 Probing turbulence with 3-dimensional particle-tracking velocimetry Exp. Therm. Fluid Sci. 4 601-12

Keane R D and Adrian R J 1992 Theory of cross-correlation analysis of PIV images Appl. Sci. Res. 49 191-215

Keane R D, Adrian R J and Zhang Y 1995 Super-resolution particle image velocimetry Meas. Sci. Technol. 6 754-68

Kohonen T 1989 Self-Organization and Associative Memory (New York: Springer)

Labonté G 1999 A new neural network for particle-tracking velocimetry Exp. Fluids 26 340-6

Labonté G 2000 On a neural network that performs an enhanced nearest-neighbour matching Pattern Anal. Applic. 3 267-78

Malik N A and Dracos T 1993 Lagrangian PTV in 3D flows Appl. Sci. Res. 51 161-6

Malik N A, Dracos T and Papantoniou D A 1993 Particle tracking velocimetry in three-dimensional flows: II. Particle tracking Exp. Fluids 15 279-94

Ohmi K and Lam D H 1998 New particle tracking PIV using an improved relaxation method Proc. 8th Int. Symp. on Flow Visualization (Sorrento, Italy) pp 1-8

Ohmi K and Li H Y 2000 Particle-tracking velocimetry with new algorithms Meas. Sci. Technol. 11 603-16

Okamoto K, Schmidl W D and Hassan Y A 1995 Least force technique for the particle tracking algorithm Flow Visualization VII ed J P Crowder (New York: Begell House) pp 647-53

Pereira F and Gharib M 2002 Defocusing digital particle image velocimetry and the three-dimensional characterization of two-phase flows Meas. Sci. Technol. 13 683-94

Pereira F and Gharib M 2004 A method for three-dimensional particle sizing in two-phase flows Meas. Sci. Technol. 15 2029-38

Racca R G and Dewey J M 1988 A method for automatic particle tracking in a three-dimensional flow field Exp. Fluids 6 25-32

Ruhnau P, Guetter C, Putze T and Schnörr C 2005 A variational approach for particle tracking velocimetry Meas. Sci. Technol. 16 1449-58

Shan J and Gharib M 1998 Super-resolution particle tracking velocimetry Proc. Int. Symp. on Flow Visualization (Seattle, WA)

Stitou A and Riethmuller M L 2001 Extension of PIV to super resolution using PTV Meas. Sci. Technol. 12 1398-403

Stüer H 1999 Investigation of separation on a forward facing step PhD Thesis ETH Zürich, Switzerland, no 13132

Wernet M P and Pline A 1993 Particle displacement tracking technique and Cramer-Rao lower-bound error in centroid estimates from CCD imagery Exp. Fluids 15 295-307

Willert C E and Gharib M 1991 Digital particle image velocimetry Exp. Fluids 10 181-93 